\title{
The phylogenetic position of enteromonads: a challenge for the present models of diplomonad evolution
}

\author{
Martin Kolisko, Ivan Cepicka, Vladimír Hampl, Jaroslav Kulda \\ and Jaroslav Flegr \\ Department of Parasitology, Faculty of Science, Charles University, Prague, Czech Republic
}

Correspondence

Martin Kolisko

martin.kolisko@seznam.cz

\begin{abstract}
Unikaryotic enteromonads and diplokaryotic diplomonads have been regarded as closely related protozoan groups. It has been proposed that diplomonads originated within enteromonads in a single event of karyomastigont duplication. This paper presents the first study to address these questions using molecular phylogenetics. The sequences of the small-subunit rRNA genes for three isolates of enteromonads were determined and a tree constructed with available diplomonad, retortamonad and Carpediemonas sequences. The diplomonad sequences formed two main groups, with the genus Giardia on one side and the genera Spironucleus, Hexamita and Trepomonas on the other. The three enteromonad sequences formed a clade robustly situated within the diplomonads, a position inconsistent with the original evolutionary proposal. The topology of the tree indicates either that the diplokaryotic cell of diplomonads arose several times independently, or that the monokaryotic cell of enteromonads originated by secondary reduction from the diplokaryotic state.
\end{abstract}

\section{INTRODUCTION}

The protozoan groups Retortamonadida, Diplomonadida and Enteromonadida have been regarded as closely related (Brugerolle, 1991; Corliss, 1994). The presence of a feeding groove or cytostome associated with the posterior flagellum places diplomonads, enteromonads and retortamonads among the suggested monophylum of excavates (Simpson \& Patterson, 1999; Simpson, 2003). In his recent taxonomic revision, Cavalier-Smith (2003) places these three groups into the superclass Eopharyngia of the infrakingdom Excavata. The monophyly of the Excavata is, however, controversial and has little support in molecular data (Simpson et al., 2002; Simpson, 2003).

The most prominent characteristic of the order Diplomonadida is a duplicated karyomastigont - two nuclei, each associated with four basal bodies, microtubular fibres and a cytostome, if present. The presumed phylogenetic affinity of diplomonads to unikaryotic retortamonads has been confirmed by molecular phylogenetic studies (Silberman et al., 2002; Simpson et al., 2002). In these analyses,

Published online ahead of print on 14 March 2005 as DOI 10.1099/ ijs.0.63542-0.

Abbreviation: SSU, small subunit.

The GenBank/EMBL/DDBJ accession numbers for the small-subunit rRNA sequences of enteromonad isolates KR-PO3, RAPI1 and TRION are AY701872, AY701873 and AY701874, respectively. retortamonads constituted a sister clade of the diplomonad genus Giardia, causing the paraphyly of diplomonads. However, the statistical support for this topology was rather low, and morphological data (e.g. karyomastigont organization) strongly suggest the monophyly of Giardia with other diplomonads (Simpson, 2003). The phylogenetic position of enteromonads has never been studied using molecular methods. The organization of the enteromonad cytoskeleton is very similar to that of diplomonads, apart from the fact that the structures are not duplicated. The trophozoite of enteromonads can be described, in simplified fashion, as one half of the cell of a hexamitid diplomonad. There are three known genera of enteromonads - Enteromonas, Trimitus and Caviomonas - inhabiting the intestines of both vertebrates and invertebrates. Enteromonas hominis is a facultative human pathogen (Spriegel et al., 1989; Goldberg, 1990). On the basis of ultrastructural data, Brugerolle (1975) created, from enteromonads and diplomonads, two suborders (Diplomonadina and Enteromonadina) of a single order (Diplomonadida). Brugerolle also proposed the phylogenetic scheme of diplomonad evolution, in which enteromonads constituted a sister group of diplomonads. In a cladistic study based on morphological characteristics (Siddall et al., 1992), the genera Enteromonas and Trimitus constituted a paraphyletic group at the root of the diplomonad clade. This led Siddall et al. (1992) to a hypothesis that the single karyomastigont of enteromonads was a plesiomorphic state and the double karyomastigont of 
diplomonads arose by its duplication in a single event. The authors also proposed the mechanism of this transformation - heterochrony, i.e. a change in the relative timing of cytokinesis and karyokinesis. In a recent study (Simpson, 2003), the morphological characteristics did not provide sufficient resolution in the phylogeny of excavate protists. The position of Enteromonas and Trimitus was more or less unresolved, but different from the position in Siddall's cladogram. They branched as a sister clade of Hexamita and Spironucleus or as separated branches within diplomonads.

In the present work, we employed, for the first time, molecular methods to resolve the phylogenetic position of enteromonads using the small-subunit (SSU) rRNA sequences of three isolates of enteromonads. We discuss the implications of our results on the hypotheses of the origins of unikaryotic and diplokaryotic cells within the diplomonad-enteromonad-retortamonad clade.

\section{METHODS}

Isolation and cultivation. Two isolates of Trimitus sp. were obtained from the intestine of the turtle Aspideretes gangeticus (strain TRION) and from the cloaca of the frog Rana pipiens (strain RAPI1). These isolates were maintained in xenic (agnothobiotic) cultures in Dobell-Leidlaw's biphasic medium (Dobell \& Leidlaw, 1926). Both isolates showed morphology typical of the genus, as demonstrated by microscopic observations of specimens stained with Protargol (Bayer) (Fig. 1). Trimitus sp. was the only eukaryotic organism present in the isolate TRION. Two eukaryotic organisms, enteromonad Trimitus sp. and trichomonad Trichomitus sp., were present in the isolate RAPI1. Another enteromonad isolate, KR-PO3, which failed to grow in subcultures, was obtained from the faeces of

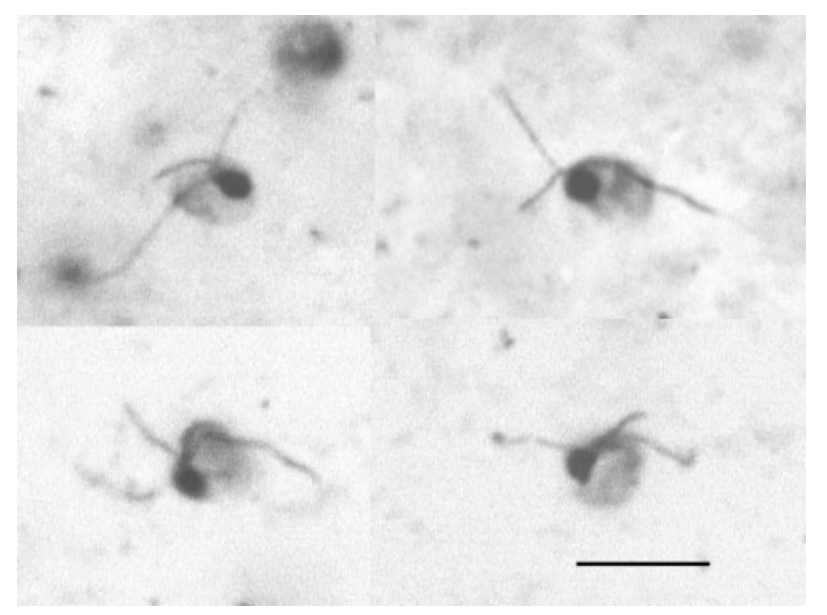

Fig. 1. Trophozoites of Trimitus sp., isolate TRION, from the turtle $A$. gangeticus (Trionychidae), showing the characteristic morphology of the genus. The cell is equipped with two unequal anterior flagella and one posterior flagellum that is adherent, in part, to the cell surface and continues in the form of a free trailing portion. Protargol silver stain was used. Bar, $5 \mu \mathrm{m}$. cattle. The TRION and RAPI1 isolates are deposited in the culture collection of the Department of Parasitology of Charles University, Prague, Czech Republic.

DNA amplification. Genomic DNA was isolated using the High Pure PCR template kit (Roche Applied Science). Primers $5^{\prime}$ end primer A and $3^{\prime}$ end primer B (Medlin et al., 1988) were used to amplify the SSU rRNA gene from the genomic DNA of the TRION strain. The major PCR fragment $(1500 \mathrm{bp})$ was purified from gel and subcloned. Two clones with $1500 \mathrm{bp}$ inserts from two independent PCRs were sequenced bidirectionally by primer walking. Two Trimitus-specific primers were designed on the basis of the sequence obtained: EntUnvF (5'-CGTCAAAGATTAAAACATGCATAT-3') and EntUnvR (5'-TCCTCTAAGCCTTCTAGTTCGTGCAAA-3'). Using these specific primers, the SSU rRNA genes of the RAPI1 and KR-PO3 strain cells were amplified, subcloned and sequenced. The SSU rRNA genes of all of the enteromonad isolates were also amplified and sequenced directly from PCR products.

Phylogenetic analyses. All sequences of the SSU rRNA genes of diplomonads available in public databases, except for the incomplete sequence of Spironucleus muris, were used in our analyses. Representatives of most major eukaryotic groups were used as outgroups (25 sequences). The alignment based on secondary structure was downloaded from the rRNA server (http://www.psb.ugent.be/rRNA/). Sequences of SSU rRNA of three isolates of enteromonads, Spironucleus barkhanus, Spironucleus vortens, six retortamonads and Carpediemonas membranifera were appended to the downloaded alignment and realigned using the function 'realign selected sequences' with the program CLUSTAL X 1.18 (Thompson et al., 1997). The alignment obtained was manually edited using the program BIOEDIT (Hall, 1999). The final alignment contained 1043 positions. Phylogenetic trees were constructed using maximumlikelihood, Bayesian, maximum-parsimony and distance methods in the programs PAUP 4.0b10 (Swofford, 1999), MrBayes 3.0 (Huelsenbeck, 2000) and PHYML (Guindon \& Gascuel, 2003). The model for maximum likelihood $(\operatorname{TrN}+\mathrm{I}+\Gamma)$ was chosen using the hierarchical nested likelihood ratio test implemented in MODELTEST 3.06 (Posada \& Crandall, 1998). Maximum-likelihood trees were constructed with the program PAUP, using a heuristic search (addseq $=$ random, nrep $=10, \quad$ Pinvar $=0 \cdot 1763, \quad \alpha=0.7154$ ngammacat $=4$, TBR branch swapping algorithm), and bootstrapping was performed with the program PHYML, using 100 replicates. A Bayesian tree was independently constructed three times in the program MrBayes 3.0 (ngen $=2000000$, nst $=6$, basefreq $=$ estimate, rates $=$ invgamma, burnin $=1000$ ). All three runs of MrBayes produced the same topology. Maximum-parsimony trees (addseq= random, nrep $=10$, TBR branch swapping algorithm) and leastsquares distance trees using logdet distance (Pinvar $=0 \cdot 304885$, addseq $=$ random, nrep $=10$, TBR branch swapping algorithm) were constructed using PAUP, and bootstrap analyses were performed with 1000 replicates.

\section{RESULTS AND DISCUSSION}

\section{Phylogenetic analyses}

A phylogenetic tree was constructed on the basis of SSU rRNA sequences from three isolates of enteromonads, 12 sequences of diplomonads, six sequences of retortamonads and outgroups from major eukaryotic lineages (Fig. 2a). A sister group of the Eopharyngia clade was formed either by C. membranifera (Bayesian, maximumlikelihood, maximum-parsimony methods) or by Trichomonas vaginalis (distance method). The diplomonads split 
(a)

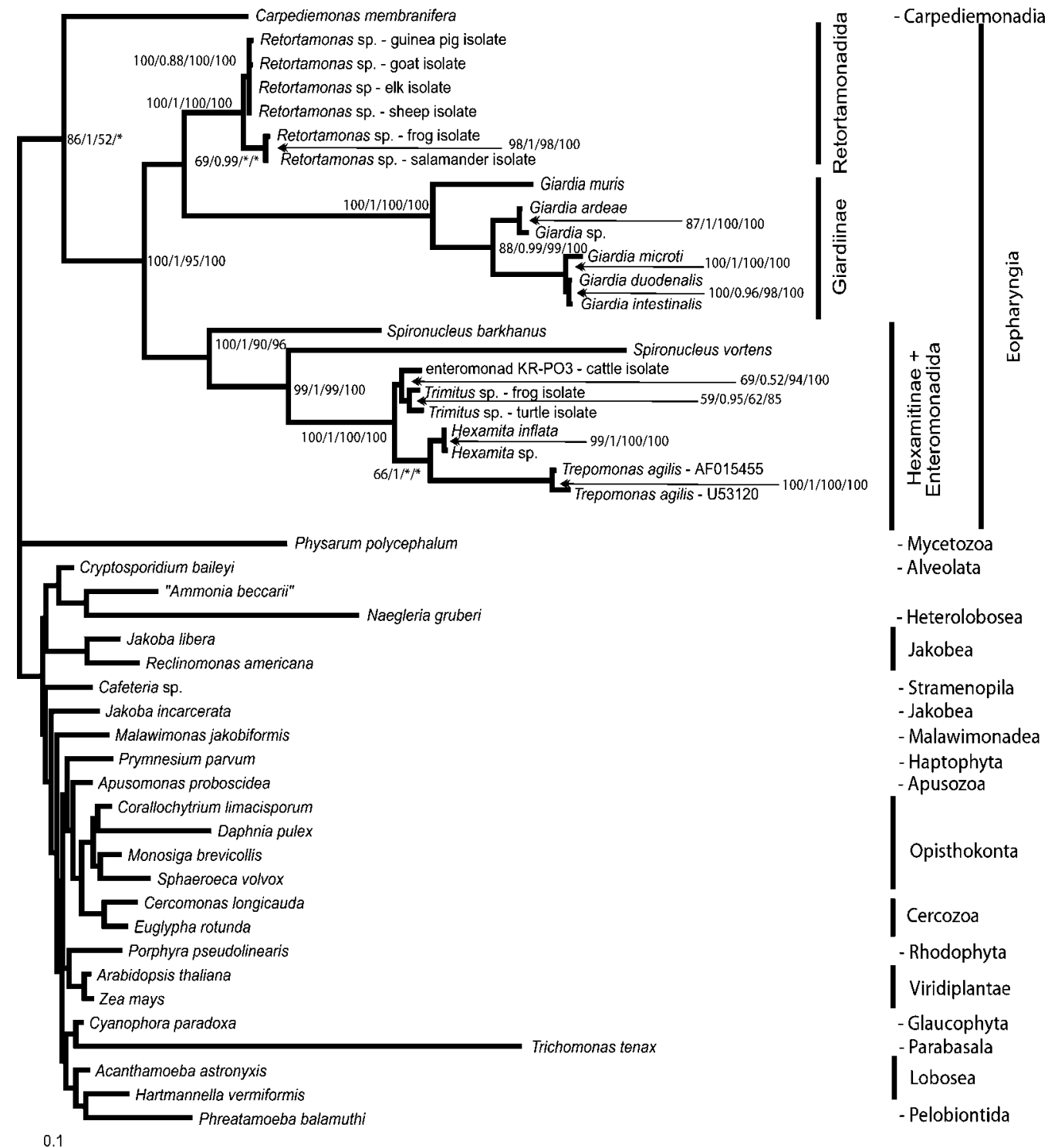

(b)

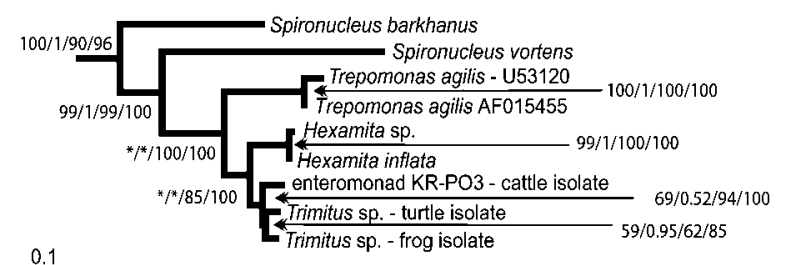

(c)

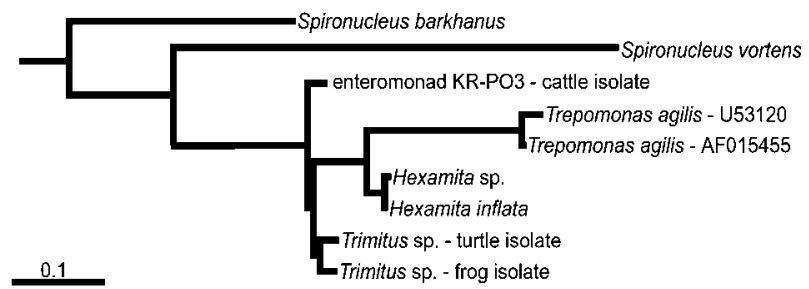

Fig. 2. (a) Phylogenetic tree of diplomonads, enteromonads and retortamonads, rooted by outgroups from the main eukaryotic lineages, constructed by using maximum likelihood. Statistical support for nodes is shown as: PHYML bootstraps/MrBayes posterior probability value/maximum parsimony bootstraps/distance method bootstraps. Bootstrap support is not quoted for outgroup taxa. Asterisks indicate that the branching was different using that construction method. (b) Topology obtained by using maximumparsimony and distance methods. (c) Topology obtained by using maximum likelihood in programs PAUP and PHYML, when no bootstrap analysis was performed. Bootstrap analysis provided the same topology as in (a). 
into two branches in accordance with their classification (Giardiinae, Hexamitinae). All tree-construction methods placed the enteromonads in a common branch with Trepomonas and Hexamita, with the highest statistical support. The topology within this clade varied with the treeconstruction methods (Fig. 2a-c). All tree-construction methods, except for the maximum-likelihood analysis, recovered a clade of enteromonads. In the maximumlikelihood analysis, enteromonad isolate KR-PO3 branched at the base of the Hexamita-Trepomonas-enteromonad subtree (Fig. 2c). The majority of trees from the maximumlikelihood bootstrap analysis also reconstructed enteromonads as a monophylum.

The genus Spironucleus constituted a paraphyletic group at the base of the Hexamitinae subtree. This result corresponds to a previous analysis (Morin, 2000) and has high statistical support. However, this branching could be artificial and should be confirmed using data from other genes.

The relationship between the three main clades - retortamonads, Giardia and Hexamita-Trepomonas-enteromonads - was not robustly resolved. Maximum-likelihood and Bayesian analyses placed retortamonads as a sister group of genus Giardia causing a paraphyly of diplomonads. On the other hand, maximum-parsimony and distance analyses recovered the diplomonad clade.

\section{The phylogenetic position of enteromonads}

The position of enteromonads in the tree clearly shows their close relationship with the genera Hexamita and Trepomonas. However, the position of enteromonads within the Hexamita-Trepomonas-enteromonads clade and the monophyly of enteromonads were not robustly supported, and various tree-construction methods produced conflicting topologies. In all trees, unikaryotic enteromonads branched above three diplokaryotic clades (Giardia, S. barkhanus and $S$. vortens) with the highest statistical support. The diplokaryotic Trepomonas and Hexamita formed a sister clade to the enteromonads in most trees. This topology suggests that the simplest and rather intuitive hypothesis of a single diplomonad origin from enteromonad ancestors does not hold. In principle, two scenarios can explain the distribution of the mono- and diplomonad morphology within the phylogenetic tree. The first scenario supposes that the last common ancestor of diplomonads and enteromonads had a double karyomastigont and that the single karyomastigont of enteromonads is evolutionary apomorphy. The second scenario holds that the single karyomastigont represents a plesiomorphic state of the group and that the double karyomastigont of diplomonads is an evolutionary apomorphy. If the position of enteromonads in the tree is correct, the second scenario would expect that the double karyomastigont of diplomonads originated four times during evolution. The number of changes of single karyomastigont to double karyomastigont could be lower, if Spironucleus constituted a clade. At face value, the first scenario, with a single origin of double karyomastigont and its reduction in enteromonads, seems to be more parsimonious. However, the reduction of the double karyomastigont to the single karyomastigont seems to be less feasible than the opposite process. It has already been suggested that the double karyomastigont of diplomonad cells could have arisen as a result of heterochrony, i.e. by the changing of the relative timing of cytokinesis and karyokinesis (Siddall et al., 1992). This hypothesis proposes that the double karyomastigont of diplomonads originated by secondary karyokinesis after the delay or arrest of cytokinesis. The resulting cell with four karyomastigonts could then divide into two cells with doubled karyomastigonts (Siddall et al., 1992). Indeed, trophozoites of Enteromonas and Trimitus with double karyomastigonts that probably originated because of delayed or arrested cytokinesis have been observed already in natural populations (Brugerolle, 1975, 1986; Kulda \& Nohýnková, 1978). Conversely, the opposite phenomenon 'cytokinesis without karyokinesis' - is necessary for the origin of a unikaryotic cell from a diplokaryotic cell. There are currently no published examples of the existence of this process, but the exact cytological mechanisms of cell and nucleus division of diplomonads are very poorly understood and it is possible that the reduction of karyomastigonts can occur during encystation or excystation processes. Therefore the possibility of the origin of a unikaryotic cell from a diplokaryotic cell cannot be excluded, so more detailed studies on the phylogeny and cell biology of diplomonads are needed to elucidate this interesting feature of diplomonad evolutionary history.

The position of Giardia depended on the tree-construction method. It branched either as a sister group of Retortamonas or as a sister group of other diplomonads [as in previous studies published by Silberman et al. (2002) and Simpson et al. (2002)]. The statistical support of both positions was low. It is clear that definitive resolution around these nodes must await more data from other genes.

Our data clearly show that none of the proposed classifications (Brugerolle, 1975; Cavalier-Smith, 2003), which assume that diplomonads and enteromonads form sister groups, reflect correctly the phylogenetic relationship of these taxa. However, before a general revision of these classifications can be attempted, more molecular and morphological data are necessary. In particular, the important question as to whether all enteromonad genera form a clade within the diplomonad branch should be answered.

\section{ACKNOWLEDGEMENTS}

We thank Alastair G. B. Simpson, Andrew J. Roger and anonymous reviewers for valuable discussions and/or comments. We also thank Nick Stuart for English correction. This work was supported by grants from the Czech Ministry of Education (grant 0021620828), the Czech Science Foundation (206/05/0371) and Grant Agency of Charles University (209/2005/B-BIO/PRF). 


\section{REFERENCES}

Brugerolle, G. (1975). Etude ultrastructurale du genre Enteromonas da Fonseca (Zoomastigophorea) et révision de l'Ordre des Diplomonadida Wenyon. J Protozool 22, 468-475 (in French).

Brugerolle, G. (1986). Séparation des genres Trimitus (Diplomonadida) and Tricercomitus (Trichomonadida) d'après leur ultrastructure. Protistologica 22, 31-37 (in French).

Brugerolle, G. (1991). Flagellar and cytoskeletal systems in amitochondrial flagellates - Archamoeba, Metamonada and Parabasala. Protoplasma 164, 70-90.

Cavalier-Smith, T. (2003). The excavate protozoan phyla Metamonada Grassé emend. (Anaeromonadea, Parabasalia, Carpediemonas, Eopharyngia) and Loukozoa emend. (Jakobea, Malawimonas): their evolutionary affinities and new higher taxa. Int J Syst Evol Microbiol 53, 1741-1758.

Corliss, J. O. (1994). An interim utilitarian ("user-friendly") hierarchical classification and characterization of the protists. Acta Protozool 33, 1-51.

Dobell, C. \& Leidlaw, P. P. (1926). On the cultivation of Entamoeba histolytica and some other parasitic amoebae. Parasitology 18, 283-318.

Goldberg, J. (1990). Enteromonas hominis incidence and diarrhea. Am J Gastroenterol 84, 480.

Guindon, S. \& Gascuel, O. (2003). A simple, fast, and accurate algorithm to estimate large phylogenies by maximum likelihood. Syst Biol 52, 696-704.

Hall, T. A. (1999). BioEdit: a user-friendly biological sequence alignment editor and analysis program for Windows 95/98/NT. Nucleic Acids Symp Ser 41, 95-98.

Huelsenbeck, J. P. (2000). MrBayes: Bayesian inference of phylogeny. Distributed by the author. Department of Biology, University of Rochester, Rochester, NY, USA.

Kulda, J. \& Nohýnková, E. (1978). Flagellates of the human intestine and of intestines of other species. In Parasitic Protozoa, vol. 2, pp. 1-138. Edited by J. P. Kreier. New York: Academic Press.
Medlin, L., Elwood, H. J., Stickel, S. \& Sogin, M. L. (1988). The characterization of enzymatically amplified eukaryotic 16S-like rRNA-coding regions. Gene 71, 491-499.

Morin, L. (2000). Long branch attraction effects and the status of "basal eukaryotes": phylogeny and structural analysis of the ribosomal RNA gene cluster of the free-living diplomonad Trepomonas agilis. J Eukaryot Microbiol 47, 167-177.

Posada, D. \& Crandall, C. A. (1998). MODELTEST: testing the model of DNA substitution. Bioinformatics 14, 817-818.

Siddall, M. E., Hong, H. \& Desser, S. S. (1992). Phylogenetic analysis of the Diplomonadida (Wenyon, 1926) Brugerolle, 1975: evidence for heterochrony in protozoa and against Giardia lamblia as a "missing link". J Protozool 39, 361-367.

Silberman, J. D., Simpson, A. G. B., Kulda, J., Cepicka, I., Hampl, V., Johnson, P. J. \& Roger, A. J. (2002). Retortamonad flagellates are closely related to diplomonads - implications for the history of mitochondrial function in eukaryote evolution. Mol Biol Evol 19, 777-786.

Simpson, A. G. B. (2003). Cytoskeletal organization, phylogenetic affinities and systematics in the contentious taxon Excavata (Eukaryota). Int J Syst Evol Microbiol 53, 1759-1777.

Simpson, A. G. B. \& Patterson, D. J. (1999). The ultrastructure of Carpediemonas membranifera (Eukaryota) with reference to the "Excavate hypothesis". Eur J Protistol 35, 353-370.

Simpson, A. G. B., Roger, A. J., Silberman, J. D., Leipe, D. D., Edgcomb, V. P., Jermiin, L. S., Patterson, D. J. \& Sogin, M. L. (2002). Evolutionary history of "early-diverging" eukaryotes: the excavate taxon Carpediemonas is a close relative of Giardia. Mol Biol Evol 19, 1782-1791.

Spriegel, J. R., Saag, K. G. \& Tsang, T. K. (1989). Infectious diarrhea secondary to Enteromonas hominis. Am J Gastroenterol 84, 1313-1314.

Swofford, D. L. (1999). PAUP*. Phylogenetic analysis using parsimony (* and other methods). Version 4.b10. Sunderland, MA: Sinauer Associates.

Thompson, J. D., Gibson, T. J., Plewniak, F., Jeanmougin, F. \& Higgins, D. G. (1997). The CLUSTAL_X Windows interface: flexible strategies for multiple sequence alignment aided by quality analysis tools. Nucleic Acids Res 25, 4876-4882. 